\title{
Reduced Basis Method for Real Time Multiple Heat Source Placement Optimization in Ablation Cancer Therapy *
}

\author{
Zoi Tokoutsi * Martin Grepl ${ }^{* *}$ Karen Veroy ${ }^{* * *}$ \\ Marco Baragona ${ }^{* * * *}$ Alfonso Isola ${ }^{* * *}$ Ralph Maessen ${ }^{* * * *}$ \\ * Philips Research, High Tech Campus 4, 5656 AE, Eindhoven, NL \\ (e-mail: tokoutsi@aices.rwth-aachen.de). \\ ** IGPM, RWTH Aachen University, Templergraben 55, 52062, \\ Aachen DE, (e-mail: grepl@igpm.rwth-aachen.de) \\ *** AICES, RWTH Aachen University, Schinkelstr. 2, 52066 Aachen \\ DE, (e-mail: veroy@aices.rwth-aachen.de) \\ **** Philips Research, High Tech Campus 4, 5656 AE, Eindhoven, $N L$
}

Keywords: Reduced-order models, Reduced basis method, Optimal control, Parameter optimization, Greedy optimization algorithm.

\section{INTRODUCTION}

Thermal ablation treatments in cancer therapy heat a target volume, enough to cause it to burn, but leave healthy tissue and neighboring sensitive structures undamaged; see Chu and Dupuy (2014). The placement of the heat source and the control of its power are essential for an effective ablation, and are affected by the size and location of the target. In the following, we propose a novel approach to the power-placement problem by dissecting it into two parts.

In the first part we determine the optimal heat source by solving a distributed elliptic optimal control problem, which is parametrized with respect to problem relevant parameters. Using the certified reduced basis method for parametrized distributed elliptic optimal control problems presented in Kärcher et al. (2017), a reliable and real-time efficient surrogate model is created.

The second part determines a reproduction of the optimal heat from the first part using heat sources produced by ablation devices. This is achieved by the optimization of the power settings and placement parameters of one or multiple heat sources. A greedy multiple heat source placement algorithm is introduced, so that multiple heat sources can be positioned in order to improve the approximation of the target function.

\section{PART I: OPTIMAL HEAT DISTRIBUTION}

\subsection{Transfer of Heat in Living Tissue}

The Pennes bioheat equation in Pennes (1948) describes the heat transfer in living tissue and approximates the cooling effect of blood circulation as an isotropic heat sink, proportional to the blood flow rate and the difference

\footnotetext{
^ This work is supported by the European Commission through the Marie Sklodowska-Curie Actions (European Industrial Doctorate, Project Nr. 642445).
}

between the body core temperature and the local tissue temperature. The non-dimensional stationary bioheat equation is

$$
\begin{array}{rlrl}
-k \Delta y+c y & =u, & & \text { in } \Omega \\
k \nabla_{\nu} y+h y=0 & & \text { on } \Gamma
\end{array}
$$

where $y$ and $u \in U=L^{2}(\Omega)$ are the temperature and heat functions, $k$ is the thermal conductivity, $c$ is the blood perfusion parameter and $h$ is the convection parameter.

The corresponding weak formulation is

$$
\begin{aligned}
\int_{\Omega} k \nabla y \nabla \phi+\int_{\Omega} c y \phi+\int_{\Gamma} h y \phi & =\int_{\Omega} u \phi \\
\Leftrightarrow a(y, \phi ; \mu) & =b(u, \phi ; \mu)
\end{aligned}
$$

for all $\phi \in Y=H^{1}(\Omega)$, where $a(\cdot, \cdot ; \mu): Y \times Y \rightarrow$ $\mathbb{R}$ is continuous and coercive, $b(\cdot, \cdot ; \mu): U \times Y \rightarrow \mathbb{R}$ is continuous, and $\mu \in \mathcal{D}$ represents the parameters of interest. Here, $\mu$ may include tissue parameters or geometric parameters such as the proximity of the tumor to risk structures as in Tokoutsi et al. (2017).

\subsection{The Distributed Optimal Heat Problem}

The optimal heat is the solution of a distributed optimal control problem, constrained by the bioheat equation (1). The computational domain is divided into target $\Omega_{1}$, risk $\Omega_{2}$ and healthy $\Omega_{3}$ tissue. The target temperature is set to be 0.18 over the target $\Omega_{1}$ and 0 elsewhere. Each term in the cost functional is weighted according to its significance. The optimal heat can be parametrized with respect to the problem parameters, and the weights of the summands of the corresponding cost functional.

The optimal heat $u^{*}$ is determined as the solution to

$$
\begin{aligned}
\min _{u \in U} J(y, u): & =\sum_{i=1}^{3} \frac{\lambda_{i}}{2}\left\|y-y_{d}\right\|_{L^{2}\left(\Omega_{i}\right)}^{2}+\frac{\lambda}{2}\|u\|_{L^{2}(\Omega)}^{2} \\
& =d\left(y-y_{d}, y-y_{d} ; \mu\right)+\lambda c(u, u ; \mu) / 2 \\
\text { s.t. } a(y, v ; \mu) & =b(u, v ; \mu) \forall v \in Y=H^{1}(\Omega)
\end{aligned}
$$


Using the Lagrangian formulation and calculating the first order optimality conditions of the resulting optimization problem, a system of equations is obtained; see e.g. Tröltzsch (2005). Given $\mu \in \mathcal{D}$ the optimal solution $\left(y^{*}, u^{*}, p^{*}\right) \in Y \times U \times Y$ satisfies

$$
\begin{array}{ll}
a\left(y^{*}, \phi ; \mu\right)=b\left(u^{*}, \phi ; \mu\right) & \forall \phi \in Y, \\
a\left(\varphi, p^{*} ; \mu\right)=d\left(y_{d}(\mu)-y^{*}, \varphi ; \mu\right) & \forall \varphi \in Y, \\
\lambda c\left(u^{*}, \psi ; \mu\right)-b\left(\psi, p^{*} ; \mu\right)=0 & \forall \psi \in U .
\end{array}
$$

Here $p^{*}$ is the adjoint function and the equations (4) correspond to the vanishing directional derivatives of the Lagrangian of (3), and are called the state, adjoint and gradient equations.

\section{REDUCED BASIS APPROXIMATION}

The efficient and reliable online solution of PART I is achieved by employing the Reduced Basis (RB) method. Using an adjusted version of the well established greedy sampling algorithm, a sample set $\mathcal{D}_{N}=\left\{\mu^{1}, \cdots, \mu^{N}\right\} \subset$ $\mathcal{D}$, the associated integrated reduced basis space $Y_{N}=$ $\operatorname{span}\left\{y^{*}\left(\mu^{n}\right), p^{*}\left(\mu^{n}\right), 1 \leq n \leq N\right\}$, and the reduced basis control space $U_{N}=\operatorname{span}\left\{u^{*}\left(\mu^{n}\right), \quad 1 \leq n \leq N\right\}, 1 \leq$ $N \leq N_{\max }$, are generated. The greedy algorithm utilizes rigorous and (online-)efficient a posteriori error bounds, which are obtained by manipulating the error residual equations of the optimality system (4). It can be shown that:

Proposition 1. For any $\mu \in \mathcal{D}$ the optimal heat error in the energy heat norm $\|\cdot\|_{U(\mu)}$ satisfies

$$
\Delta_{N}^{u, \mathrm{ALT}}(\mu):=c_{1}(\mu)+\sqrt{c_{1}(\mu)^{2}+c_{2}(\mu)},
$$

where $c_{1}(\mu), c_{2}(\mu)$ depend on the lower bound of the coercivity constant of $a(\cdot, \cdot ; \mu)$, the upper bound of the continuity constant of $b(\cdot, \cdot ; \mu)$, and the upper bound $C_{D}^{\mathrm{UB}}$ for the constant $C_{D}(\mu):=\sup _{u \in Y}|u|_{D(\mu)} /\|v\|_{Y} \geq 0$, and the dual norms of the residuals of the equations in system 4; for details, see Kärcher et al. (2017).

\section{PART II: GREEDY MULTIPLE HEAT SOURCE PLACEMENT}

\subsection{Heat Source Power-Placement Optimization}

The second part of our proposed method is concerned with the optimization of the power $P \in \mathbb{R}$ and placement $\chi \in \mathbb{R}^{m}, m=5$ in 3 -D, of a finite number of heat sources using the optimal heat $u^{*}$ of the first part as target. We assume here that the heat sources are reproducible with state-of-the-art medical equipment. We further assume that each heat source can be described using a sufficiently smooth closed formulation, e.g.

$$
Q(x, \chi, P)=P \exp \left(-(x-\chi)^{2} /\left(2 \gamma^{2}\right)\right),
$$

where the variance $\gamma^{2}$ is fitted so that $Q$ approximates the heat source produced by a radiofrequency ablation probe. The optimal placement $\chi^{\star}$ and power $P^{\star}$ of each heat source results from solving an optimization problem, where the target heat function $U=U(x ; \mu)$ depends on $u^{*}(x ; \mu)$

$$
\left(\chi^{\star}, P^{\star}\right)=\underset{\chi, P}{\operatorname{argmin}} I(\chi, P ; \mu):=\|Q(\chi, P)-U\|_{L^{2}(\Omega)}^{2}
$$

The power-placement optimization problem (6) is a low dimensional optimization problem which can be solved with common optimization algorithms such as quasi-Newton or trust region methods; see e.g. Nocedal and Wright (2006). Due to the non-convexity of the cost functional, there exist multiple local minima to $I(\chi, P ; \mu)$.

\subsection{Greedy Multiple Heat Source Placement Algorithm}

The placement of multiple heat sources can be achieved iteratively until the collective heat source $Q^{\star}$ adequately approximates the optimal heat $u^{*}$. The proposed greedy multiple heat source placement algorithm will locate the most significant local minima of $I(\chi, P ; \mu)$.

Algorithm 1. (Multiple Heat Source Placement). The algorithm is initialized with $U=u^{*}$ of PART I as target function. On each iteration $(k)$, the termination criteria are checked, the target heat source is updated to $U=U-$ $Q\left(\mu^{(k-1), \star}\right)$ and $P^{(k), \star}, \chi^{(k), \star}$ are determined by solving (6).

The termination criteria consist of the achievement of a prescribed tolerance for the relative heat error norm or for the change in the value of the cost function. A further relevant criterion refers to exceeding a prescribed maximum number of heat sources.

\section{SUMMARY}

This work presents an algorithm for multiple heat source placement, motivated by thermal ablation treatments, and our steps toward real-time efficiency using the reduced basis method. Numerical results are presented to show the performance of the proposed approach.

\section{REFERENCES}

Chu, K.F. and Dupuy, D.E. (2014). Thermal ablation of tumours: biological mechanisms and advances in therapy. Nature reviews. Cancer, 14 3, 199-208.

Kärcher, M., Tokoutsi, Z., Grepl, M.A., and Veroy, K. (2017). Certified reduced basis methods for parametrized elliptic optimal control problems with distributed controls. Journal of Scientific Computing.

Nocedal, J. and Wright, S. (2006). Numerical Optimization. Springer Series in Operations Research and Financial Engineering. Springer New York.

Pennes, H.H. (1948). Analysis of tissue and arterial blood temperatures in the resting human forearm. Journal of Applied Physiology, 1(2), 93-122.

Tokoutsi, Z., Kärcher, M., Grepl, M., Veroy, K., Baragona, M., and Maessen, R. (2017). Real time optimization of thermal ablation cancer treatments. In progress.

Tröltzsch, F. (2005). Optimale Steuerung partieller Differentialgleichungen: Theorie, Verfahren und Anwendungen. Vieweg. 